\title{
On Uniform f-vectors of Cutsets in the Truncated Boolean Lattice
}

\author{
Béla Bajnok \\ Gettysburg College \\ Gettysburg, PA 17325 USA \\ Shahriar Shahriari \\ Pomona College \\ Claremont, CA 91711 USA
}

August 20, 1997

\begin{abstract}
Let $[n]=\{1,2, \ldots, n\}$ and let $2^{[n]}$ be the collection of all subsets of $[n]$ ordered by inclusion. $\mathcal{C} \subseteq 2^{[n]}$ is a cutset if it meets every maximal chain in $2^{[n]}$, and the width of $\mathcal{C} \subseteq 2^{[n]}$ is the minimum number of chains in a chain decomposition of $\mathcal{C}$. Fix $0 \leq m \leq l \leq n$. What is the smallest value of $k$ such that there exists a cutset that consists only of subsets of sizes between $m$ and $l$, and such that it contains exactly $k$ subsets of size $i$ for each $m \leq i \leq l$ ? The answer, which we denote by $g_{n}(m, l)$, gives a lower estimate for the width of a cutset between levels $m$ and $l$ in $2^{[n]}$. After using the Kruskal-Katona Theorem to give a general characterization of cutsets in terms of the number and sizes of their elements, we find lower and upper bounds (as well as some exact values) for $g_{n}(m, l)$.
\end{abstract}

\section{Introduction}

Let $2^{[n]}$ be the Boolean lattice of order $n$, that is the lattice of all subsets (often called nodes) of $[n]=\{1,2, \ldots, n\}$ ordered by inclusion. For $0 \leq m \leq n$ we define the $m$-th level set $\left(\begin{array}{c}{[n]} \\ m\end{array}\right)$ of $2^{[n]}$ as the set of all subsets of size $m$. The $f$-vector (or profile) $\mathbf{f}=\left(f_{0}, f_{1}, \ldots, f_{n}\right)$ of a collection of subsets $\mathcal{A} \subseteq 2^{[n]}$ is defined by $f_{m}=\left|\mathcal{A}_{m}\right|$ where $\mathcal{A}_{m}=\mathcal{A} \cap\left(\begin{array}{c}{[n]} \\ m\end{array}\right)$ and $0 \leq m \leq n$.

A collection of $l+1$ subsets $A_{0} \subset A_{1} \subset \cdots \subset A_{l}$ in $2^{[n]}$ is called a chain of length $l$. A maximal chain in $2^{[n]}$ is one that has length $n$. A collection of $w$ nodes with the property that none of them contains another is called an antichain of size $w$. The length and the width of a collection of subsets $\mathcal{A} \subseteq 2^{[n]}$ are defined as the length of the longest chain and the size of the largest antichain in $\mathcal{A}$, respectively.

A cutset in $2^{[n]}$ is defined as a collection of subsets $\mathcal{C} \subseteq 2^{[n]}$ which intersects all maximal chains. Trivially, every collection $\mathcal{C}$ which contains $\emptyset$ or $[n]$ is a cutset. In 3 . we proved that for $n \geq 2$, the width of a cutset in the Boolean lattice of order $n$ which does not contain $\emptyset$ or $[n]$ is greater than or equal to $n-1$, and that for $n \geq 3$ there exist cutsets of width $n-1$ in $2^{[n]}$. Thus, it is possible to construct a cutset in $2^{[n]}$ with $f$-vector $(0, \underbrace{n-1, n-1, \ldots, n-1}_{n-1}, 0)$. We then may ask for the smallest value of $k$ for which there is a cutset in $2^{[n]}$ with $f$-vector $(0, \underbrace{k, k, \ldots, k}_{n-1}, 0)$. The original goal of our work was to show that this value is $n-2$ (see Corollary 3 below). 
More generally, for $0 \leq m \leq l \leq n$ we define $g_{n}(m, l)$ to be the smallest value of $k$ for which the $n+1$-tuple $\left(f_{0}, f_{1}, \ldots, f_{n}\right)$, defined by $f_{i}=k$ if $m \leq i \leq l$ and 0 otherwise, can be the $f$-vector of a cutset in $2^{[n]}$. Thus our goal above is then to find $g_{n}(1, n-1)$. Note that by symmetry we have $g_{n}(m, l)=g_{n}(n-l, n-m)$, so we may assume without loss of generality that $m \leq l \leq n-m$.

Before studying $g_{n}(m, l)$, we give a general characterization of $f$-vectors of cutsets in $2^{[n]}$. For a given profile $\mathbf{f}=\left(f_{0}, f_{1}, \ldots, f_{n}\right)$ and integer $m_{0}$ with $0 \leq m_{0} \leq n$, we construct a canonical collection of subsets $\mathcal{C}\left(\mathbf{f}, m_{0}\right)$, with the property that there is a cutset in $2^{[n]}$ with profile $\mathbf{f}$ if and only if $\mathcal{C}\left(\mathbf{f}, m_{0}\right)$ is a cutset for some (or every) $0 \leq m_{0} \leq n$. We then translate this qualitative criterion to a quantitative one: For a given $\mathbf{f}=\left(f_{0}, f_{1}, \ldots, f_{n}\right)$ and $0 \leq m_{0} \leq n$, we describe an easily computable value $q\left(\mathbf{f}, m_{0}\right)$, so that $\mathbf{f}$ will be the profile of a cutset in $2^{[n]}$ exactly when $f_{m_{0}} \geq q\left(\mathbf{f}, m_{0}\right)$ for some (or every) $0 \leq m_{0} \leq n$. These characterizations, which we present in Section 2, are essentially due to Daykin [7] (for a correction see [5] and then [4]), though we follow a treatment which is more suitable for our purposes.

We can then determine the values of $g_{n}(m, l)$ for $l \leq m+2$. Namely, we prove the following.

Theorem 1 Let $n$ be a positive integer.

1. $g_{n}(m, m)=\left(\begin{array}{c}n \\ m\end{array}\right)$ for every integer $0 \leq m \leq n$.

2. $g_{n}(m, m+1)=\left(\begin{array}{c}n-1 \\ m\end{array}\right)$ for every integer $0 \leq m \leq n-1$.

3. $g_{n}(m, m+2)=\sum_{j=0}^{m}\left(\begin{array}{c}n-2 j-2 \\ m-j\end{array}\right)$ for every integer $0 \leq m \leq n / 2-1$.

Next, viewing $m$ as fixed and $n>>m$ (i.e., for all $n>n_{0}=n_{0}(m)$ ), we develop upper and lower bounds for $g_{n}(m, l)$.

Theorem 2 Suppose that $m$ and $n$ are non-negative integers and $n>>m$. Then

1. $\left(\begin{array}{c}n-2 \\ m\end{array}\right)<g_{n}(m, l) \leq \sum_{j=0}^{m}\left(\begin{array}{c}n-2 j-2 \\ m-j\end{array}\right)$ for every integer $m+2 \leq l \leq n-m-1$, and

2. $\left(\begin{array}{c}n-3 \\ m\end{array}\right)<g_{n}(m, n-m) \leq \sum_{j=0}^{m}\left(\begin{array}{c}n-2 j-3 \\ m-j\end{array}\right)$.

For $m=1$ we then get the following results.

Corollary 3 Suppose that $n>4$ and $1 \leq l \leq n-1$ are integers. Then

$$
g_{n}(1, l)= \begin{cases}n & \text { if } l=1 \\ n-1 & \text { if } 2 \leq l \leq n-2 \\ n-2 & \text { if } l=n-1\end{cases}
$$

For $2 \leq m<<n$, Theorems 1 and 2 give the "numerator" of the leading term of the $m$-binomial representation (see section 2) of $g_{n}(m, l)$. Namely, this value is equal to $n$ if $l=m, n-1$ if $l=m+1$, $n-2$ if $m+2 \leq l \leq n-m-1$, and $n-3$ if $l=n-m$. It is striking that for a rather large range of values of $l, g_{n}(m, l)$ stays essentially unchanged. 
We note that in Theorem 2, the ratio of the upper bound to the lower bound is approximately $1+\frac{m}{n}$, and thus the bounds are rather accurate as $n>>m$.

Extremal problems regarding cutsets in the Boolean lattice have been the object of much study. For example see [8, 10, 12, 13, 15, 17, 18].

\section{$2 f$-vectors of cutsets}

Given a collection $\mathcal{B} \subseteq\left(\begin{array}{c}{[n]} \\ m\end{array}\right)$, the shadow and the shade of $\mathcal{B}$ will be denoted by $\triangle \mathcal{B}$ and $\nabla \mathcal{B}$, respectively [1, Chapter 2], and are as usual defined by

$$
\begin{aligned}
& \triangle \mathcal{B}=\left\{A \in\left(\begin{array}{c}
{[n]} \\
m-1
\end{array}\right) \mid A \subseteq B \text { for some } B \in \mathcal{B}\right\} \\
& \nabla \mathcal{B}=\left\{A \in\left(\begin{array}{c}
{[n]} \\
m+1
\end{array}\right) \mid B \subseteq A \text { for some } B \in \mathcal{B}\right\}
\end{aligned}
$$

We order the elements of $\left(\begin{array}{c}{[n]} \\ m\end{array}\right)$ by the squashed order (also called the colex order) 11, Chapter 7], that is for $A, B \in\left(\begin{array}{c}{[n]} \\ m\end{array}\right)$, we say $A<_{S} B$ if the largest element of the symmetric difference of $A$ and $B$ is in $B$. For $1 \leq K \leq\left(\begin{array}{c}n \\ m\end{array}\right)$, we define the initial collection $\mathcal{F}_{m}(K)$ and the last collection $\mathcal{L}_{m}(K)$ at level $m$ as the first and last $K$ elements in the squashed order at level $m$, respectively. In addition, if $K \leq 0$, then $\mathcal{F}_{m}(K)=\mathcal{L}_{m}(K)=\emptyset$. The squashed order has the property that the shadow of an initial collection at level $m$ is an initial collection at level $m-1$, and the shade of a last collection at level $m$ is a last collection at level $m+1$. The Kruskal-Katona Theorem ([14, 16] or [1, Chapter 7]) states that the size of the shadow of $K$ nodes at level $m$ is greater than or equal to the size of the shadow of $\mathcal{F}_{m}(K)$ and, equivalently, the size of their shade is greater than or equal to the size of the shade of $\mathcal{L}_{m}(K)$.

Let $\Omega_{n}$ denote the set of $n+1$-tuples of integers $\left(a_{0}, a_{1}, \ldots, a_{n}\right)$ such that $0 \leq a_{m} \leq\left(\begin{array}{l}n \\ m\end{array}\right)$ for all $0 \leq m \leq n$. To see whether a given $\mathbf{f} \in \Omega_{n}$ is the profile of a cutset in $2^{[n]}$, we construct a collection of subsets $\mathcal{C}=\mathcal{C}\left(\mathbf{f}, m_{0}\right)$, called the canonical collection of subsets for profile $\mathbf{f}$ and for level $m_{0}\left(0 \leq m_{0} \leq n\right)$. As we show below, there is a cutset in $2^{[n]}$ with profile $\mathbf{f}$ if and only if this canonical collection is a cutset for some (or every) $m_{0}$.

Our construction is as follows. First we let $\mathcal{E}_{0}^{\uparrow}=\{\emptyset\}, \mathcal{C}_{0}^{\uparrow}=\mathcal{F}_{0}\left(f_{0}\right)$, and for $1 \leq m \leq n$ we recursively define $\mathcal{E}_{m}^{\uparrow}=\nabla\left(\mathcal{E}_{m-1}^{\uparrow}-\mathcal{C}_{m-1}^{\uparrow}\right)$ and $\mathcal{C}_{m}^{\uparrow}=\mathcal{L}_{m}\left(\left|\mathcal{E}_{m}^{\uparrow}\right|\right)-\mathcal{L}_{m}\left(\left|\mathcal{E}_{m}^{\uparrow}\right|-f_{m}\right)$. Then $\mathcal{E}_{m}^{\uparrow}$ is a last collection at level $m$, and it is precisely the set of nodes from which there is a chain of length $m$ to $\emptyset$ which is disjoint from $\mathcal{C}_{i}^{\uparrow}$ for all $0 \leq i \leq m-1$. Analogously, we let $\mathcal{E}_{n}^{\downarrow}=\{[n]\}, \mathcal{C}_{n}^{\downarrow}=\mathcal{L}_{n}\left(f_{n}\right)$, and for $0 \leq m \leq n-1$ we recursively define $\mathcal{E}_{m}^{\downarrow}=\triangle\left(\mathcal{E}_{m+1}^{\downarrow}-\mathcal{C}_{m+1}^{\downarrow}\right)$ and $\mathcal{C}_{m}^{\downarrow}=\mathcal{F}_{m}\left(\left|\mathcal{E}_{m}^{\downarrow}\right|\right)-\mathcal{F}_{m}\left(\left|\mathcal{E}_{m}^{\downarrow}\right|-f_{m}\right)$. This time $\mathcal{E}_{m}^{\downarrow}$ is an initial collection at level $m$, and it is the set of nodes from which there is a chain of length $n-m$ to $[n]$ which is disjoint from $\mathcal{C}_{i}^{\uparrow}$ for all $m+1 \leq i \leq n$. Finally, we define $\mathcal{C}=\mathcal{C}\left(\mathbf{f}, m_{0}\right)=\left(\cup_{i=0}^{m_{0}} \mathcal{C}_{i}^{\uparrow}\right) \cup\left(\cup_{i=m_{0}+1}^{n} \mathcal{C}_{i}^{\downarrow}\right)$.

We can easily see that $\mathcal{C}=\mathcal{C}\left(\mathbf{f}, m_{0}\right)$ is a cutset if and only if $\mathcal{E}_{m_{0}}^{\uparrow} \cap \mathcal{E}_{m_{0}}^{\downarrow} \subseteq \mathcal{C}_{m_{0}}$. Furthermore, the profile $\left(c_{0}, c_{1}, \ldots, c_{n}\right)$ of $\mathcal{C}$ satisfies $c_{m} \leq f_{m}$ for every $m$, and if $\mathcal{C}$ is not a cutset, then its profile is exactly $\mathbf{f}$. 
For example, let $n=5$. If $\mathbf{f}=(0,2,5,6,0,0)$ then, for all $0 \leq m_{0} \leq 5, \mathcal{C}\left(\mathbf{f}, m_{0}\right)$ becomes $\{\{1\},\{2\},\{1,3\},\{2,3\},\{1,4\},\{2,4\},\{3,4\},\{1,2,5\},\{1,3,5\},\{2,3,5\},\{1,4,5\},\{2,4,5\},\{3,4,5\}\}$. On the other, hand if $\mathbf{g}=(0,2,6,5,0,0)$, then for $\mathcal{C}(\mathbf{g}, 5)$ we get

$$
\{\{1\},\{2\},\{1,3\},\{2,3\},\{1,4\},\{2,4\},\{3,4\},\{1,5\},\{1,2,5\},\{1,3,5\},\{2,3,5\},\{1,4,5\},\{2,4,5\}\} .
$$

It is easily seen that the first is a cutset, while the second one is a collection with profile $\mathbf{g}$ and not a cutset.

The next two propositions give us useful ways of determining whether a given vector $\mathbf{f} \in \Omega_{n}$ can be the profile of a cutset in $2^{[n]}$.

Proposition 4 Let $\mathbf{f} \in \Omega_{n}$ and $0 \leq m_{0} \leq n$. The canonical collection $\mathcal{C}\left(\mathbf{f}, m_{0}\right)$ defined above is a cutset if and only if $\left|\mathcal{E}_{m_{0}}^{\uparrow}\right|+\left|\mathcal{E}_{m_{0}}^{\downarrow}\right| \leq\left(\begin{array}{c}n \\ m_{0}\end{array}\right)+f_{m_{0}}$.

Proof. If $\mathcal{C}$ is a cutset, then the assertion follows as $\left|\mathcal{E}_{m_{0}}^{\uparrow}\right|+\left|\mathcal{E}_{m_{0}}^{\downarrow}\right|=\left|\mathcal{E}_{m_{0}}^{\uparrow} \cap \mathcal{E}_{m_{0}}^{\downarrow}\right|+\left|\mathcal{E}_{m_{0}}^{\uparrow} \cup \mathcal{E}_{m_{0}}^{\downarrow}\right| \leq$ $\left|\mathcal{C}_{m_{0}}\right|+\left(\begin{array}{c}n \\ m_{0}\end{array}\right) \leq f_{m_{0}}+\left(\begin{array}{c}n \\ m_{0}\end{array}\right)$.

On the other hand, if $\mathcal{C}$ is not a cutset, then its profile must be exactly f. Furthermore, as $\mathcal{E}_{m_{0}}^{\downarrow}$ is an initial segment and $\mathcal{E}_{m_{0}}^{\uparrow}$ is a last segment at level $m_{0}$, their intersection has size greater than $f_{m_{0}}$ and their union is all of $\left(\begin{array}{c}{[n]} \\ m_{0}\end{array}\right)$. Therefore, in this case we have $\left|\mathcal{E}_{m_{0}}^{\uparrow}\right|+\left|\mathcal{E}_{m_{0}}^{\downarrow}\right|=$ $\left|\mathcal{E}_{m_{0}}^{\uparrow} \cap \mathcal{E}_{m_{0}}^{\downarrow}\right|+\left|\mathcal{E}_{m_{0}}^{\uparrow} \cup \mathcal{E}_{m_{0}}^{\downarrow}\right|>f_{m_{0}}+\left(\begin{array}{c}n \\ m_{0}\end{array}\right)$.

The next proposition shows the importance of the canonical collection. It further shows that the choice of $m_{0}$ is immaterial.

Proposition 5 Let $\mathbf{f} \in \Omega_{n}$. The following are equivalent.

1. $\mathbf{f}$ is the profile of a cutset in $2^{[n]}$.

2. $\mathcal{C}\left(\mathbf{f}, m_{0}\right)$ is a cutset for some $0 \leq m_{0} \leq n$.

3. $\mathcal{C}\left(\mathbf{f}, m_{0}\right)$ is a cutset for every $0 \leq m_{0} \leq n$.

Proof. Clearly, if $\mathcal{C}\left(\mathbf{f}, m_{0}\right)$ is a cutset for some $0 \leq m_{0} \leq n$, then there is a cutset in $2^{[n]}$ with profile $\mathbf{f}$. Therefore, it is enough to prove that if there is an $\mathcal{A} \subseteq 2^{[n]}$ which is a cutset with profile f, then $\mathcal{C}\left(\mathbf{f}, m_{0}\right)$ is a cutset for every $0 \leq m_{0} \leq n$.

For $m=0, \ldots n$, let $\mathcal{A}_{m}=\{A \in \mathcal{A}|| A \mid=m\}$. We define $\mathcal{B}_{m}^{\uparrow}$ and $\mathcal{B}_{m}^{\downarrow}$ recursively by $\mathcal{B}_{0}^{\uparrow}=\{\emptyset\}, \mathcal{B}_{m}^{\uparrow}=\nabla\left(\mathcal{B}_{m-1}^{\uparrow}-\mathcal{A}_{m-1}\right)$ for $m=1,2, \ldots, n$, and $\mathcal{B}_{n}^{\downarrow}=\{[n]\}, \mathcal{B}_{m}^{\downarrow}=\triangle\left(\mathcal{B}_{m+1}^{\downarrow}-\mathcal{A}_{m+1}\right)$ for $m=0,1, \ldots, n-1$. Since $\mathcal{A}$ is a cutset, we must have $\mathcal{B}_{m}^{\downarrow} \cap \mathcal{B}_{m}^{\uparrow} \subseteq \mathcal{A}_{m}$ for every $0 \leq m \leq n$.

Keeping the notations established prior to Proposition 4, we now use downward induction on $m$ to prove that $\left|\mathcal{B}_{m}^{\downarrow}\right| \geq\left|\mathcal{E}_{m}^{\downarrow}\right|$ and $\left|\mathcal{B}_{m}^{\uparrow}\right| \geq\left|\mathcal{E}_{m}^{\uparrow}\right|$ for every $0 \leq m \leq n$. Clearly $\left|\mathcal{B}_{n}^{\downarrow}\right| \geq\left|\mathcal{E}_{n}^{\downarrow}\right|$. Using, in order, the definition of $\mathcal{B}_{m}^{\downarrow}$, the Kruskal-Katona theorem, the triangle inequality, the inductive 
hypothesis, the fact that $\mathcal{C}_{m+1}^{\downarrow} \subseteq \mathcal{E}_{m+1}^{\downarrow}$, that $\mathcal{E}_{m+1}^{\downarrow}-\mathcal{C}_{m+1}^{\downarrow}$ is an initial segment at level $m+1$, and the definition of $\mathcal{E}_{m}^{\downarrow}$, we can write

$$
\begin{aligned}
\left|\mathcal{B}_{m}^{\downarrow}\right| & =\left|\triangle\left(\mathcal{B}_{m+1}^{\downarrow}-\mathcal{A}_{m+1}\right)\right| \\
& \geq\left|\triangle \mathcal{F}_{m+1}\left(\left|\mathcal{B}_{m+1}^{\downarrow}-\mathcal{A}_{m+1}\right|\right)\right| \\
& \geq\left|\triangle \mathcal{F}_{m+1}\left(\left|\mathcal{B}_{m+1}^{\downarrow}\right|-\left|\mathcal{A}_{m+1}\right|\right)\right| \\
& \geq\left|\triangle \mathcal{F}_{m+1}\left(\left|\mathcal{E}_{m+1}^{\downarrow}\right|-\left|\mathcal{C}_{m+1}^{\downarrow}\right|\right)\right| \\
& =\left|\triangle \mathcal{F}_{m+1}\left(\left|\mathcal{E}_{m+1}^{\downarrow}-\mathcal{C}_{m+1}^{\downarrow}\right|\right)\right| \\
& =\left|\triangle\left(\mathcal{E}_{m+1}^{\downarrow}-\mathcal{C}_{m+1}^{\downarrow}\right)\right| \\
& =\left|\mathcal{E}_{m}^{\downarrow}\right|
\end{aligned}
$$

as claimed. The assertion for $\left|\mathcal{B}_{m}^{\uparrow}\right|$ can be proved similarly.

Our assertion now follows from Proposition 4, as

$$
\left|\mathcal{E}_{m_{0}}^{\uparrow}\right|+\left|\mathcal{E}_{m_{0}}^{\downarrow}\right| \leq\left|\mathcal{B}_{m_{0}}^{\uparrow}\right|+\left|\mathcal{B}_{m_{0}}^{\downarrow}\right|=\left|\mathcal{B}_{m_{0}}^{\uparrow} \cap \mathcal{B}_{m_{0}}^{\downarrow}\right|+\left|\mathcal{B}_{m_{0}}^{\uparrow} \cup \mathcal{B}_{m_{0}}^{\downarrow}\right| \leq\left|\mathcal{A}_{m_{0}}\right|+\left|\mathcal{B}_{m_{0}}^{\uparrow} \cup \mathcal{B}_{m_{0}}^{\downarrow}\right| \leq f_{m_{0}}+\left(\begin{array}{c}
n \\
m_{0}
\end{array}\right) .
$$

A quantitative version of Proposition [5 can be formulated as follows.

Given positive integers $K$ and $m$, there exist unique integers $a_{m}>a_{m-1}>\cdots>a_{t} \geq t \geq 1$ such that

$$
K=\left(\begin{array}{c}
a_{m} \\
m
\end{array}\right)+\left(\begin{array}{c}
a_{m-1} \\
m-1
\end{array}\right)+\cdots+\left(\begin{array}{c}
a_{t} \\
t
\end{array}\right)
$$

This is called the $m$-binomial representation of $K$ [1, Theorem 7.2.1]. Using the $m$-binomial representation of $K$ it is easy to describe the set numbered $K$ in the squashed order on the $m$-th level of the Boolean lattice [1, page 117].

For each positive integer $m$ we define a (boundary) operator $\partial_{m}$ [1] ([6] and [9] have other notations) on the integers as follows: If $K$ is a positive integer with an $m$-binomial representation as above then

$$
\partial_{m}(K)=\left(\begin{array}{c}
a_{m} \\
m-1
\end{array}\right)+\left(\begin{array}{c}
a_{m-1} \\
m-2
\end{array}\right)+\cdots+\left(\begin{array}{c}
a_{t} \\
t-1
\end{array}\right)
$$

and for non-positive $K$ set $\partial_{m}(K)=0$. Note that $\partial_{m}$ is weakly increasing.

With this operator we can write $\triangle \mathcal{F}_{m}(K)=\mathcal{F}_{m-1}\left(\partial_{m}(K)\right)$, and $\nabla \mathcal{L}_{m}(K)=\mathcal{L}_{m+1}\left(\partial_{n-m}(K)\right)$, and the Kruskal-Katona theorem becomes $|\triangle \mathcal{B}| \geq \partial_{m}(|\mathcal{B}|)$ and $|\nabla \mathcal{B}| \geq \partial_{n-m}(|\mathcal{B}|)$ where B is a collection of subsets of $[n]$ at level $m$.

Theorem 6 For a given $\mathbf{f}=\left(f_{0}, f_{1}, \ldots, f_{n}\right) \in \Omega_{n}$, define $\mathbf{u}(\mathbf{f})=\left(u_{0}, u_{1}, \ldots, u_{n}\right)$ and $\mathbf{v}(\mathbf{f})=$ $\left(v_{0}, v_{1}, \ldots, v_{n}\right)$ by:

$$
\begin{aligned}
& u_{0}=1 \text { and } u_{m+1}=\partial_{n-m}\left(u_{m}-f_{m}\right) \text { for } m=0, \ldots, n-1, \\
& v_{n}=1 \text { and } v_{m-1}=\partial_{m}\left(v_{m}-f_{m}\right) \text { for } m=1, \ldots, n .
\end{aligned}
$$

Then the following are equivalent. 
1. $\mathbf{f}$ is the profile of a cutset in $2^{[n]}$.

2. $u_{m}+v_{m}-f_{m} \leq\left(\begin{array}{c}n \\ m\end{array}\right)$ for some $0 \leq m \leq n$.

3. $u_{m}+v_{m}-f_{m} \leq\left(\begin{array}{c}n \\ m\end{array}\right)$ for every $0 \leq m \leq n$.

Proof. $\left(u_{0}, u_{1}, \ldots, u_{n}\right)$ and $\left(v_{0}, v_{1}, \ldots, v_{n}\right)$ were chosen so that $\left|\mathcal{E}_{m}^{\uparrow}\right|=u_{m}$ and $\left|\mathcal{E}_{m}^{\downarrow}\right|=v_{m}$, so the statements follow from Propositions 4 and 5 .

Note that, as a special case when $f_{0}=f_{n}=0, \mathbf{f}$ is the profile of a cutset in $2^{[n]}$ if and only if $v_{0}=0$ (or if and only if $u_{n}=0$ ).

Remark. We can give a quantitative description of the canonical collection $\mathcal{C}\left(\mathbf{f}, m_{0}\right)=\left(\cup_{i=0}^{m_{0}} \mathcal{C}_{i}^{\uparrow}\right) \cup$ $\left(\cup_{i=m_{0}+1}^{n} \mathcal{C}_{i}^{\downarrow}\right)$ as follows. If levels of $2^{[n]}$ are in the squashed order, then $\mathcal{C}_{i}^{\uparrow}$ is the segment at level $i$ starting with node numbered $\left(\begin{array}{c}n \\ i\end{array}\right)-u_{i}+1$ and ending with $\min \left\{\left(\begin{array}{c}n \\ i\end{array}\right)-u_{i}+f_{i},\left(\begin{array}{c}n \\ i\end{array}\right)\right\} ; \mathcal{C}_{i}^{\downarrow}$ is the segment at level $i$ starting with node numbered $\max \left\{1, v_{i}-f_{i}+1\right\}$ and ending with $v_{i}$.

\section{Exact values: Proof of Theorem 1}

We will use Theorem [6 to determine the values of $g_{n}(m, l)$ for $l=m, m+1$, and $m+2$.

The case $l=m$ is obvious. For $l=m+1$, we can easily see that the canonical collection with profile $\mathbf{f}=\left(0,0, \ldots, 0,\left(\begin{array}{c}n-1 \\ m\end{array}\right),\left(\begin{array}{c}n-1 \\ m\end{array}\right), 0, \ldots, 0\right)$ (where the nonzero components occur at levels $m$ and $m+1$ ) consists of subsets of size $m$ that do not contain $n$, together with subsets of size $m+1$ that contain $n$. This collection is a cutset. On the other hand, the canonical collection with profile $\mathbf{f}^{\prime}=\left(0,0, \ldots, 0,\left(\begin{array}{c}n-1 \\ m\end{array}\right),\left(\begin{array}{c}n-1 \\ m\end{array}\right)-1,0, \ldots, 0\right)$ is not a cutset as there exists a maximal chain through the node $\{n-m, n-m+1, \ldots, n\}$ which does not intersect the collection.

For the case $l=m+2$, the result is trivial for $m=0$ and hence assume that $m \geq 1$. Define $f=$ $\sum_{j=0}^{m-1}\left(\begin{array}{c}n-2 j-2 \\ m-j\end{array}\right)$, and we need to prove that $g_{n}(m, l)=f+1$. We will show that $\mathbf{f}=(0,0, \ldots, 0, f+$ $1, f, f, 0, \ldots, 0)$ (where the nonzero components occur at levels $m, m+1$, and $m+2$ ) is the profile of a cutset, but that $\mathbf{f}^{\prime}=(0,0, \ldots, 0, f, f, f, 0, \ldots, 0)$ is not the profile of a cutset.

Before starting the proof we need to establish a binomial identity which establishes a relationship between two vertical columns in the arithmetic (a.k.a. Pascal's) triangle. Let $n$ be a positive integer, let $1 \leq m \leq n / 2$, and let $0 \leq d \leq m-1$. Using downward induction on $d$ (with base case $d=m-1$ ) it can be easily shown that

$$
\sum_{j=0}^{m-d-1}\left(\begin{array}{c}
n-2 j-2 \\
m-j
\end{array}\right)+\sum_{j=0}^{m-d-1}\left(\begin{array}{c}
n-2 j-1 \\
m+2-j
\end{array}\right)+\left(\begin{array}{c}
n-2 m+2 d \\
d+2
\end{array}\right)=\left(\begin{array}{c}
n \\
m+2
\end{array}\right) .
$$

In our computations below we rely on the case $d=0$ of this identity, namely:

$$
f+\sum_{j=0}^{m-1}\left(\begin{array}{c}
n-2 j-1 \\
m+2-j
\end{array}\right)+\left(\begin{array}{c}
n-2 m \\
2
\end{array}\right)=\left(\begin{array}{c}
n \\
m+2
\end{array}\right)
$$

We now use Theorem 6 to show that $\mathbf{f}$ is the profile of a cutset. Computing $\mathbf{v}(\mathbf{f})$ we get 


$$
\begin{aligned}
& v_{i}=\left(\begin{array}{c}
n \\
i
\end{array}\right) \quad \text { for } i=n, n-1, \ldots, m+2, \\
& v_{m+1}=\partial_{m+2}\left[\left(\begin{array}{c}
n \\
m+2
\end{array}\right)-f\right] \\
&=\partial_{m+2}\left[\sum_{j=0}^{m-1}\left(\begin{array}{c}
n-2 j-1 \\
m+2-j
\end{array}\right)+\left(\begin{array}{c}
n-2 m \\
2
\end{array}\right)\right] \\
&=\sum_{j=0}^{m-1}\left(\begin{array}{c}
n-2 j-1 \\
m+1-j
\end{array}\right)+\left(\begin{array}{c}
n-2 m \\
1
\end{array}\right), \\
& v_{m}=\partial_{m+1}\left[\sum_{j=0}^{m-1}\left(\begin{array}{c}
n-2 j-1 \\
m+1-j
\end{array}\right)+\left(\begin{array}{c}
n-2 m \\
1
\end{array}\right)-f\right] \\
&=\partial_{m+1}\left[\sum_{j=0}^{m-1}\left(\begin{array}{c}
n-2 j-2 \\
m+1-j
\end{array}\right)+\left(\begin{array}{c}
n-2 m \\
1
\end{array}\right)\right] \\
&=\sum_{j=0}^{m-1}\left(\begin{array}{c}
n-2 j-2 \\
m-j
\end{array}\right)+\left(\begin{array}{c}
n-2 m \\
0
\end{array}\right) \\
&=f+1, \quad \text { for } i=m-1, m-2, \ldots, 0, \\
&=0 \\
& v_{m-1}[f+1-f-1] \\
&= 0, \quad \text { and }
\end{aligned}
$$

hence $\mathbf{f}$ is the profile of a cutset. As $\mathbf{f}^{\prime}$ differs from $\mathbf{f}$ only in its $m$-th coordinate, we still have $v_{m}=f+1$. Continuing, we get

$$
\begin{aligned}
v_{m-1} & =\partial_{m}[f+1-f] \\
& =\partial_{m}\left[\left(\begin{array}{l}
m \\
m
\end{array}\right)\right] \\
& =\left(\begin{array}{c}
m \\
m-1
\end{array}\right), \text { and } \\
v_{i} & =\left(\begin{array}{c}
m \\
i
\end{array}\right) \quad \text { for } i=m-1, m-2, \ldots, 0 .
\end{aligned}
$$

In particular, $v_{0}=1$, so $\mathbf{f}^{\prime}$ is not the profile of a cutset.

Remark. We can also construct a (non-canonical) cutset with $f$-vector $\mathbf{f}=(0,0, \ldots, 0, f+$ $1, f, f, 0, \ldots, 0)$ where $f=\sum_{j=0}^{m-1}\left(\begin{array}{c}n-2 j-2 \\ m-j\end{array}\right)$. We define $\mathcal{Q}_{0}=\left(\begin{array}{c}n-2] \\ m\end{array}\right)$ and for $j=1,2, \ldots, m$ we let

$$
\mathcal{Q}_{j}=\left\{A \cup\{n-1, n-3, \ldots, n-2 j+1\} \mid A \in\left(\begin{array}{c}
{[n-2 j-2]} \\
m-j
\end{array}\right)\right\},
$$




$$
\begin{aligned}
\mathcal{R}_{j} & =\left\{Q \cup\{n-2 j+2\} \mid Q \in \mathcal{Q}_{j-1}\right\}, \text { and } \\
\mathcal{S}_{j} & =\left\{R \cup\{n-2 j+1\} \mid R \in \mathcal{R}_{j-1}\right\} .
\end{aligned}
$$

Then it is rather straight forward to check that $\mathcal{Q}=\bigcup_{j=0}^{m} \mathcal{Q}_{j}, \mathcal{R}=\bigcup_{j=1}^{m} \mathcal{R}_{j}$, and $\mathcal{S}=\bigcup_{j=1}^{m} \mathcal{S}_{j}$ are collections of nodes at levels $m, m+1$, and $m+2$, respectively, $|\mathcal{Q}|=f+1,|\mathcal{R}|=f$, and $|\mathcal{S}|=f$, and that $\mathcal{Q} \cup \mathcal{R} \cup \mathcal{S}$ is a cutset, thus providing a cutset with $f$-vector $\mathbf{f}=(0,0, \ldots, 0, f+1, f, f, 0, \ldots, 0)$. We omit the details but only briefly sketch the idea behind the construction: Note that $\mathcal{Q}_{0}$ consists of all subsets of size $m$ that do not contain $n$ or $n-1$. Likewise, $\mathcal{R}_{1}$ consists of all subsets of size $m+1$ that contain $n$ but not $n-1$ and $\mathcal{S}_{1}$ consists of all subsets of size $m+2$ that contain $n$ and $n-1$. Thus any maximal chain in $2^{[n]}$ that does not intersect $\mathcal{Q}_{0} \cup \mathcal{R}_{1} \cup \mathcal{S}_{1}$ will have subsets of size $m, m+1$ and $m+2$ that contain $n-1$ but not $n$. Now the poset of subsets of $[n]$ that contain $n-1$ but not $n$ is isomorphic (as a poset) to $2^{[n-2]}$. We now restrict ourself to this poset and continue the construction recursively.

\section{Bounds: Proof of Theorem 2}

We need the following propositions.

Proposition 7 For $m \leq l<n / 2$ we have $g_{n}(m, n-m) \leq g_{n-1}(m, l)$.

Proof. Let $\mathcal{A} \subseteq 2^{[n-1]}$ be a cutset with $f$-vector $(0, \ldots, 0, g, \ldots, g, 0, \ldots, 0)$, where $g=g_{n-1}(m, l)$ and the nonzero entries are at levels $m \leq i \leq l$. Let $\overline{\mathcal{A}}=\{[n] \backslash A \mid A \in \mathcal{A}\}$. Note that by symmetry $\overline{\mathcal{A}}$ is a cutset in the poset $\mathcal{Q}$ consisting of all subsets of $[n]$ that contain $n$. Now define $\mathcal{B}=\mathcal{A} \cup \overline{\mathcal{A}}$. Note that since $l<n-l, \mathcal{B}$ has $f$-vector $(0, \ldots, 0, g, \ldots, g, 0, \ldots, 0, g, \ldots, g, 0, \ldots, 0)$, where the nonzero entries are at levels $m \leq i \leq l$ and $n-l \leq i \leq n-m$. It now suffices to show that $\mathcal{B}$ is a cutset in $2^{[n]}$.

Let $\mathcal{C}=C_{0} \subset C_{1} \subset \cdots \subset C_{n}$ be a maximal chain in $2^{[n]}$ with $\left|C_{i}\right|=i$ for $0 \leq i \leq n$, and suppose, indirectly, that $\mathcal{B} \cap \mathcal{C}=\emptyset$. Since $\mathcal{A}$ is a cutset in $2^{[n-1]}$ with support between levels $m$ and $l$, we must have $n \in C_{k}$ for every $l \leq k \leq n$. Similarly, since $\overline{\mathcal{A}}$ is a cutset in $\mathcal{Q}$, we must have $n \notin C_{k}$ for every $0 \leq k \leq n-l$. This can only happen if $n-l<l$ which is a contradiction.

Proposition 8 Suppose that $m+2 \leq l \leq n-m-1$ and $g_{n-1}(m+1, l-1)>\left(\begin{array}{c}n-2 \\ m\end{array}\right)$. Then $g_{n}(m, l)>\left(\begin{array}{c}n-2 \\ m\end{array}\right)$.

Proof. Let $\mathbf{f}=\left(0,0, \ldots, 0,\left(\begin{array}{c}n-2 \\ m\end{array}\right),\left(\begin{array}{c}n-2 \\ m\end{array}\right), \ldots,\left(\begin{array}{c}n-2 \\ m\end{array}\right), 0, \ldots, 0\right)$, where the nonzero entries occur between levels $m$ and $l$. Suppose, indirectly, that $\mathbf{f}$ is the profile of a cutset in $2^{[n]}$. Then, by Proposition 5, the canonical collection $\mathcal{C}(\mathbf{f}, n)$ is such a cutset. If $C_{i}=\mathcal{C} \cap\left(\begin{array}{c}{[n]} \\ i\end{array}\right)$ are the level sets of the collection, then it is easy to see that $C_{m}=\left(\begin{array}{c}{[n-2]} \\ m\end{array}\right)$ and $C_{m+1}=\{A \cup\{n-$ $\left.1\} \mid A \in\left(\begin{array}{c}{[n-2]} \\ m\end{array}\right)\right\}$. Therefore $\cup_{i=m+2}^{l} C_{i}$ is a (canonical) cutset in $\{A \cup\{n\} \mid A \subseteq[n-1]\}$ of profile $\left(0,0, \ldots, 0,\left(\begin{array}{c}n-2 \\ m\end{array}\right),\left(\begin{array}{c}n-2 \\ m\end{array}\right), \ldots,\left(\begin{array}{c}n-2 \\ m\end{array}\right), 0, \ldots, 0\right)$, where the nonzero entries occur between levels $m+2$ and $l$. But $\{A \cup\{n\} \mid A \subseteq[n-1]\}$ is isomorphic to $2^{[n-1]}$, so we must have $g_{n-1}(m+1, l-1) \leq\left(\begin{array}{c}n-2 \\ m\end{array}\right)$, a contradiction. 
Proposition 9 Define $\mathbf{f}=\left(f_{0}, f_{1}, \ldots, f_{n}\right)$ as follows. Let $f_{m}=f_{m+1}=f_{n-m-1}=f_{n-m}=\left(\begin{array}{c}n-3 \\ m\end{array}\right)$, $f_{i}=f_{n-i}=\left(\begin{array}{c}n+m-i-1 \\ m+1\end{array}\right)$ for $i=m+2, m+3, \ldots,\lfloor n / 2\rfloor$, and $f_{i}=0$ otherwise. Then $\mathbf{f}$ is not the profile of a cutset in $2^{[n]}$.

Proof. For v(f) we get the following.

$$
\begin{aligned}
& v_{i}=\left(\begin{array}{l}
n \\
i
\end{array}\right) \quad \text { for } \quad i=n, n-1, \ldots, n-m, \\
& v_{n-m-1}=\partial_{n-m}\left[\left(\begin{array}{c}
n \\
n-m
\end{array}\right)-\left(\begin{array}{c}
n-3 \\
n-m-3
\end{array}\right)\right] \\
& =\partial_{n-m}\left[\left(\begin{array}{c}
n-1 \\
n-m
\end{array}\right)+\left(\begin{array}{c}
n-2 \\
n-m-1
\end{array}\right)+\left(\begin{array}{c}
n-3 \\
n-m-2
\end{array}\right)\right] \\
& =\left(\begin{array}{c}
n-1 \\
n-m-1
\end{array}\right)+\left(\begin{array}{c}
n-2 \\
n-m-2
\end{array}\right)+\left(\begin{array}{c}
n-3 \\
n-m-3
\end{array}\right) \text {, } \\
& v_{n-m-2}=\partial_{n-m-1}\left[\left(\begin{array}{c}
n-1 \\
n-m-1
\end{array}\right)+\left(\begin{array}{c}
n-2 \\
n-m-2
\end{array}\right)+\left(\begin{array}{c}
n-3 \\
n-m-3
\end{array}\right)-\left(\begin{array}{c}
n-3 \\
n-m-3
\end{array}\right)\right] \\
& =\partial_{n-m-1}\left[\left(\begin{array}{c}
n-1 \\
n-m-1
\end{array}\right)+\left(\begin{array}{c}
n-2 \\
n-m-2
\end{array}\right)\right] \\
& =\left(\begin{array}{c}
n-1 \\
n-m-2
\end{array}\right)+\left(\begin{array}{c}
n-2 \\
n-m-3
\end{array}\right) \text {, } \\
& v_{n-m-3}=\partial_{n-m-2}\left[\left(\begin{array}{c}
n-1 \\
n-m-2
\end{array}\right)+\left(\begin{array}{c}
n-2 \\
n-m-3
\end{array}\right)-\left(\begin{array}{c}
n-3 \\
n-m-4
\end{array}\right)\right] \\
& =\partial_{n-m-2}\left[\left(\begin{array}{c}
n-1 \\
n-m-2
\end{array}\right)+\left(\begin{array}{c}
n-3 \\
n-m-3
\end{array}\right)\right] \\
& =\left(\begin{array}{c}
n-1 \\
n-m-3
\end{array}\right)+\left(\begin{array}{c}
n-3 \\
n-m-4
\end{array}\right) \text {, } \\
& v_{n-m-4}=\partial_{n-m-3}\left[\left(\begin{array}{c}
n-1 \\
n-m-3
\end{array}\right)+\left(\begin{array}{c}
n-3 \\
n-m-4
\end{array}\right)-\left(\begin{array}{c}
n-4 \\
n-m-5
\end{array}\right)\right] \\
& =\partial_{n-m-3}\left[\left(\begin{array}{c}
n-1 \\
n-m-3
\end{array}\right)+\left(\begin{array}{c}
n-4 \\
n-m-4
\end{array}\right)\right] \\
& =\left(\begin{array}{c}
n-1 \\
n-m-4
\end{array}\right)+\left(\begin{array}{c}
n-4 \\
n-m-5
\end{array}\right) \text {, }
\end{aligned}
$$

and continuing as above, we get $v_{k}=\left(\begin{array}{c}n-1 \\ k\end{array}\right)+\left(\begin{array}{c}k+m \\ k-1\end{array}\right)$ for every $k \geq\lfloor(n-1) / 2\rfloor$.

Now note that since $\mathbf{f}$ is symmetrical, for $\mathbf{v}(\mathbf{f})$ and $\mathbf{u}(\mathbf{f})$ we have $v_{i}=u_{n-i}$ for every $0 \leq i \leq n$. Therefore

$$
v_{\lfloor n / 2\rfloor}+u_{\lfloor n / 2\rfloor}=v_{\lfloor n / 2\rfloor}+v_{\lceil n / 2\rceil}=\left(\begin{array}{c}
n-1 \\
\lfloor n / 2\rfloor
\end{array}\right)+\left(\begin{array}{c}
\lfloor n / 2\rfloor+m \\
\lfloor n / 2\rfloor-1
\end{array}\right)+\left(\begin{array}{c}
n-1 \\
\lceil n / 2\rceil
\end{array}\right)+\left(\begin{array}{c}
\lceil n / 2\rceil+m \\
\lceil n / 2\rceil-1
\end{array}\right)=\left(\begin{array}{c}
n \\
\lfloor n / 2\rfloor
\end{array}\right)+f_{\lfloor n / 2\rfloor}+f_{\lceil n / 2\rceil},
$$
thus $\mathbf{f}$ cannot be the profile of a cutset by Theorem 6 . 
Proof of Theorem Q 2 and Corollary [3. The upper bound in 1 follows from Theorem 1 part 3 since $g_{n}(m, l)$ is weakly decreasing with $l$. The upper bound in 2 is a consequence of Proposition 7 (take $l=m+2$ assuming $n>2 m+4$ ) and Theorem 11 part 3. Proposition 9 implies that $g_{n}(m, n-m)>\min \left\{\left(\begin{array}{c}n-3 \\ m\end{array}\right),\left(\begin{array}{c}\lfloor n / 2\rfloor+m-1 \\ m+1\end{array}\right)\right\}$. It is an elementary exercise to check that this minimum is $\left(\begin{array}{c}n-3 \\ m\end{array}\right)$ for $n>>m$ (e.g. if $n \geq 3^{m+1}(m+1)$ ), establishing the lower bound in 2. Finally, the lower bound in 1 is a consequence of Proposition 8 taking $l=n-m-1$, and noting that $\left(\begin{array}{c}n-4 \\ m+1\end{array}\right) \geq\left(\begin{array}{c}n-2 \\ m\end{array}\right)$ for $n>>m$ (e.g. if $n \geq 8(m+1))$ and the lower bound in 2 .

The cases of $l=1,2$ (and 3) of Corollary 3 follow from Theorem 1 . The assertions for $3 \leq l \leq$ $n-1$ follow from Theorem 2 if $n \geq 18$ (see above). The cases $5 \leq n \leq 17$ were checked directly using Theorem 6 (and a simple computer program).

We close this section by proving a partial complement to Proposition 7 .

Proposition 10 For $n / 2-1<l \leq n-m-2$ we have $g_{n}(m, n-m) \geq g_{n-1}(m, l)$.

Proof. Consider the canonical collection $\mathcal{C}=\mathcal{C}(\mathbf{f}, l)$ where $\mathbf{f}=\left(f_{0}, f_{1}, \ldots, f_{n}\right)$ is defined by $f_{i}=g_{n}(m, n-m)$ for $m \leq i \leq n-m$ and 0 otherwise. Then $\mathcal{C}$ is a cutset in $2^{[n]}$. Write $\mathcal{D}_{1}=\{C \in \mathcal{C}|| C \mid \leq l\}$ and $\mathcal{D}_{2}=\{C \in \mathcal{C}|| C \mid \geq l+1\}$.

Suppose, indirectly, that $g_{n}(m, n-m)<g_{n-1}(m, l)$. Then $\mathcal{D}_{1}$ is not a cutset in $2^{[n-1]}$, in particular, $\mathcal{D}_{1} \subseteq 2^{[n-1]}$. Since $l \geq n-1-l$, by symmetry $\mathcal{D}_{2}$ is disjoint from $2^{[n-1]}$. Therefore $\mathcal{D}_{1} \cup \mathcal{D}_{2}=\mathcal{C}$ is not a cutset in $2^{[n]}$, a contradiction.

\section{$5 \quad$ Examples and conjectures}

The following table has the exact values of $g_{n}(m, l)$ for $n=100, m=4$ and every $4 \leq l \leq 96$. These were found using Theorem 6 . We have also given the 4-binomial representation of $g_{n}(m, l)$ in the third column.

\begin{tabular}{||c||c||c||}
\hline \hline$l$ & $g_{100}(4, l)$ & 4-binomial representation of $g_{100}(4, l)$ \\
\hline \hline 4 & $3,921,225$ & $\left(\begin{array}{c}100 \\
4\end{array}\right)$ \\
\hline 5 & $3,764,376$ & $\left(\begin{array}{c}99 \\
4\end{array}\right)$ \\
\hline 6 & $3,759,624$ & $\left(\begin{array}{c}98 \\
4\end{array}\right)+\left(\begin{array}{c}96 \\
3\end{array}\right)+\left(\begin{array}{c}94 \\
2\end{array}\right)+\left(\begin{array}{c}93 \\
1\end{array}\right)$ \\
\hline 7 & $3,759,526$ & $\left(\begin{array}{c}98 \\
4\end{array}\right)+\left(\begin{array}{c}96 \\
3\end{array}\right)+\left(\begin{array}{c}93 \\
2\end{array}\right)+\left(\begin{array}{c}88 \\
1\end{array}\right)$ \\
\hline $8 \leq l \leq 95$ & $3,759,525$ & $\left(\begin{array}{c}98 \\
4\end{array}\right)+\left(\begin{array}{c}96 \\
3\end{array}\right)+\left(\begin{array}{c}93 \\
2\end{array}\right)+\left(\begin{array}{c}87 \\
1\end{array}\right)=\left(\begin{array}{c}100 \\
4\end{array}\right)-\left(\begin{array}{c}100 \\
3\end{array}\right)$ \\
\hline 96 & $3,607,527$ & $\left(\begin{array}{c}97 \\
4\end{array}\right)+\left(\begin{array}{c}95 \\
3\end{array}\right)+\left(\begin{array}{c}92 \\
2\end{array}\right)+\left(\begin{array}{c}86 \\
1\end{array}\right)=\left(\begin{array}{c}99 \\
4\end{array}\right)-\left(\begin{array}{c}99 \\
3\end{array}\right)$ \\
\hline \hline
\end{tabular}

Theorem 1 gives the exact value for $4 \leq l \leq 6$. For $6 \leq l \leq 95$, Theorem 2 gives $3,612,280=$ $\left(\begin{array}{c}98 \\ 4\end{array}\right)<g_{100}(4, l) \leq\left(\begin{array}{c}98 \\ 4\end{array}\right)+\left(\begin{array}{c}96 \\ 3\end{array}\right)+\left(\begin{array}{c}94 \\ 2\end{array}\right)+\left(\begin{array}{c}92 \\ 1\end{array}\right)+\left(\begin{array}{c}90 \\ 0\end{array}\right)=3,759,624$. Finally, for $l=96$, Theorem 2 gives $3,464,840=\left(\begin{array}{c}97 \\ 4\end{array}\right)<g_{100}(4,96) \leq\left(\begin{array}{c}97 \\ 4\end{array}\right)+\left(\begin{array}{c}95 \\ 3\end{array}\right)+\left(\begin{array}{c}93 \\ 2\end{array}\right)+\left(\begin{array}{c}91 \\ 1\end{array}\right)+\left(\begin{array}{c}89 \\ 0\end{array}\right)=3,607,625$.

From these and other similar tables we see how $g_{n}(m, l)$ decreases as $l$ increases from $m$ to $n-m$. Namely, we observe that the decrease is largest from level $m$ to level $m+1$ and from level $n-m-1$ to level $n-m$, quite modest between level $m+2$ and level $2 m$, and that, rather strikingly, $g_{n}(m, l)$ is constant between levels $l=2 m$ and $l=n-m-1$. In fact, we have the following conjectures. 
Conjecture 11 For $n>>m$ we have

1. $g_{n}(m, l)=\left(\begin{array}{c}n \\ m\end{array}\right)-\left(\begin{array}{c}n \\ m-1\end{array}\right)$ for every $l=2 m, 2 m+1, \ldots, n-m-1$, and

2. $g_{n}(m, n-m)=\left(\begin{array}{c}n-1 \\ m\end{array}\right)-\left(\begin{array}{c}n-1 \\ m-1\end{array}\right)$.

Note that the $m$-binomial representation of $\left(\begin{array}{c}n \\ m\end{array}\right)-\left(\begin{array}{c}n \\ m-1\end{array}\right)$ starts with $\left(\begin{array}{c}n-2 \\ m\end{array}\right)+\left(\begin{array}{c}n-4 \\ m-1\end{array}\right)$ (and $\left(\begin{array}{c}n-1 \\ m\end{array}\right)-$ $\left(\begin{array}{c}n-1 \\ m-1\end{array}\right)$ starts with $\left.\left(\begin{array}{c}n-3 \\ m\end{array}\right)+\left(\begin{array}{c}n-5 \\ m-1\end{array}\right)\right)$ when $n>>m$ (cf. Theorem 2). Corollary 3 proves our conjectures for $m=1$, and Theorem 2 establishes that Conjecture 11 provides an upper bound for $m=2$ since $\left(\begin{array}{l}n \\ 2\end{array}\right)-\left(\begin{array}{l}n \\ 1\end{array}\right)=\left(\begin{array}{c}n-2 \\ 2\end{array}\right)+\left(\begin{array}{c}n-3 \\ 1\end{array}\right)\left(\right.$ and $\left.\left(\begin{array}{c}n-1 \\ 2\end{array}\right)-\left(\begin{array}{c}n-1 \\ 1\end{array}\right)=\left(\begin{array}{c}n-3 \\ 2\end{array}\right)+\left(\begin{array}{c}n-4 \\ 1\end{array}\right)\right)$.

According to Conjecture 11, we have $g_{n}(m, n-m)=g_{n-1}(m, n-m-2)=g_{n-1}(m, l)$ for $n>>m$ and $2 m \leq l \leq n-m-2$. Proposition 7 establishes $g_{n}(m, n-m) \leq g_{n-1}(m, l)$ for $l<n / 2$, while Proposition 10 proves the other direction for $n / 2-1<l \leq n-m-2$ (yielding equality when $l=(n-1) / 2)$.

Note: In a subsequent paper [2], the above conjectures have been somewhat refined and related to other conjectures about the width of cutsets in the truncated Boolean lattice.

\section{References}

[1] I. Anderson. Combinatorics of Finite Sets. Oxford University Press, Oxford, 1989.

[2] B. Bajnok. On the minimum width of a cutset in the truncated Boolean lattice. Congr. Numer., 130:77-81, 1998.

[3] B. Bajnok and S. Shahriari. Long symmetric chains in the Boolean lattice. Journal of Combinatorial Theory A, 75:44-54, 1996.

[4] G. F. Clements. Errata to: Antichains in the set of subsets of a multiset, discrete math. 48 (1984) 23-45. unpublished.

[5] G. F. Clements. Antichains in the set of subsets of a multiset. Discrete Mathematics, 48:23-45, 1984 .

[6] C. J. Colbourn. The Combinatorics of Network Reliability. Oxford University Press, Oxford, 1987.

[7] D. E. Daykin. Antichains in the lattice of subsets of a finite set. Nanta Math, 8(2):84-94, 1975.

[8] D. Duffus, B. Sands, and P. Winkler. Maximal chains and antichains in the Boolean lattices. SIAM Journal of Discrete Mathematics, 3(2):197-205, 1990.

[9] P. Frankl, M. Matsumoto, I. Z. Ruzsa, and N. Tokushige. Minimum shadows in uniform hypergraphs and a generalization of the Takagi function. Journal of Combinatorial Theory A, 69:125-148, 1995.

[10] Z. Füredi, J. R. Griggs, and D. J. Kleitman. A minimum cutset of the Boolean lattice with almost all members. Graphs and Combinatorics, 5:327-332, 1989. 
[11] C. Greene and D. J. Kleitman. Proof techniques in the theory of finite sets. In G.-C. Rota, editor, Studies in Combinatorics, volume 17 of MAA studies in Mathematics, pages 22-79. The Mathematical Association of America, 1978.

[12] J. R. Griggs. Matchings, cutsets, and chain partitions in graded posets. Discrete Mathematics, 144:33-46, 1995.

[13] J. R. Griggs and D. J. Kleitman. Minimum cutsets for an element of a Boolean lattice. Order, 6:31-37, 1989.

[14] G. O. H. Katona. A theorem on finite sets. In Theory of Graphs. Proc. Colloq. Tihany, pages 187-207. Akademiai Kiado. Academic Press, New York, 1966.

[15] D. J. Kleitman. Extremal problems of hypergraphs. In P. Frankl, Z. Füredi, G. Katona, and D. Miklós, editors, Extremal Problems for Finite Sets, pages 355-374. János Bolyai Mathematical Society, Budapest, 1994.

[16] J. B. Kruskal. The number of simplices in a complex. In R. Bellman, editor, Mathematical optimization techniques, pages 251-78. University of California Press, Berkeley, 1963.

[17] R. Nowakowski. Cutsets of Boolean lattices. Discrete Mathematics, 63:231-240, 1987.

[18] F. Shi and W. Li. The density of a maximum minimal cut in the subset lattice of a finite set is almost one. Discrete Mathematics, 123:111-115, 1993. 